\title{
Light Meson Decays at BESIII
}

\author{
Shuangshi Fang (for the BESIII Collaboration) $)^{1,}$ a \\ ${ }^{1}$ Institute of High Energy Physics, Beijing 100049, China
}

\begin{abstract}
At present the world's largest sample of 1.3 billion $J / \psi$ events was accumulated at the BESIII detector, which offers a unique place to study light meson decays. The recent results on the light meson decays are reviewed in this talk. An emphasis is put on the significant progresses on the study of $\eta / \eta^{\prime}$ decays, including Dalitz plot analysis of $\eta / \eta^{\prime} \rightarrow \pi \pi \pi$, observation of new decay modes $\left(\eta^{\prime} \rightarrow \pi^{+} \pi^{-} \pi^{+(0)} \pi^{-(0)}, \eta^{\prime} \rightarrow \rho^{ \pm} \pi^{\mp}, \eta^{\prime} \rightarrow\right.$ $\gamma e^{+} e^{-}$and $\eta^{\prime} \rightarrow e^{+} e^{-} \omega$ ), study of $\eta^{\prime} \rightarrow \gamma \pi^{+} \pi^{-}$and search for the rare decay of $\eta^{\prime} \rightarrow K \pi$. In addition, a prospect on the Dalitz plot analysis of $\omega \rightarrow \pi^{+} \pi^{-} \pi^{0}$ is presented.
\end{abstract}

\section{Introduction}

$\eta / \eta^{\prime}$ and $\omega$ are the ground states of pseudoscalar and vector nonets, respectively. Since $\eta$ and $\eta^{\prime}$ were discovered half a century ago, both of them have been attracted both theoretical and experimental attentions due to their special role in understanding low energy Quantum Chromodynamics (QCD). In particular for $\eta^{\prime}$, it is much heavier than the Goldstone bosons of broken chiral symmetry, and it has a special role in hadron physics because of its interpretation as a singlet state arising from the axial $U(1)$ anomaly. Therefore $\eta^{\prime}$ decay dynamics remains a subject of extensive theoretical studies aiming at extensions of chiral perturbation theory (ChPT). Recently a dispersive analysis of $\omega / \phi \rightarrow \pi^{+} \pi^{-} \pi^{0}$ was performed in Ref. [1, 2], and it was found that the dispersive relation could provides a good description of the data of $\phi \rightarrow \pi^{+} \pi^{-} \pi^{0}$ from KLOE experiment [3]. Since the limited statistics of $\omega$ decays, up to date the experimental Dalitz plot investigation on $\omega$ has never been performed.

In addition to the many achievements on $J / \psi$ decays and the light meson spectroscopy, the BESIII experiment also offers a unique a place to investigate the light meson decays because of their high production rate in $J / \psi$ decays. At present a sample of $1.31 \times 10^{9} \mathrm{~J} / \psi$ events [4] were accumulated at the BESIII detector, which corresponds to $1.4 \times 10^{6} \eta\left(6.8 \times 10^{6} \eta^{\prime}\right)$ after taking into account the branching fraction of $J / \psi$ radiative decays into $\eta\left(\eta^{\prime}\right)$. Though the $\omega$ meson can not be directly produced from $J / \psi$ radiative decays, the production rate for $\omega$ in $J / \psi$ hadronic decays is quite high, e.g., $B(J / \psi \rightarrow \omega \eta)=$ $(1.74 \pm 0.20) \times 10^{-3}$ [5], which allows to select a clean sample of $8 \times 10^{5} \omega$ events to investigate the dynamics of $\omega \rightarrow \pi^{+} \pi^{-} \pi^{0}$ even after considering the branching fraction of $\eta \rightarrow \gamma \gamma$. In this talk I would like made a review on the recent results on the light mesons, mainly focusing on

\footnotetext{
a e-mail: fangss@ihep.ac.cn
}

the significant progresses on $\eta / \eta^{\prime}$ decays, achieved at the BESIII experiment.

\section{$2 \eta / \eta^{\prime}$ hadronic decays}

\subsection{Observation of $\eta^{\prime} \rightarrow \pi^{+} \pi^{-} \pi^{+} \pi^{-}, \pi^{+} \pi^{-} \pi^{0} \pi^{0}$ [6]}

The hadronic decays $\eta^{\prime} \rightarrow \pi^{+} \pi^{-} \pi^{+(0)} \pi^{-(0)}$ are not suppressed by approximate symmetries. Recently Guo, Kubis and Wirzba [7], using a combination of chiral perturbation theory (ChPT) and a vector-meson dominance (VMD) model, obtained the following prediction : $\mathcal{B}\left(\eta^{\prime} \rightarrow\right.$ $\left.\pi^{+} \pi^{-} \pi^{+} \pi^{-}\right)=(1.0 \pm 0.3) \times 10^{-4}$ and $\mathcal{B}\left(\eta^{\prime} \rightarrow \pi^{+} \pi^{-} \pi^{0} \pi^{0}\right)=$ $(2.4 \pm 0.7) \times 10^{-4}$. The $\pi^{+} \pi^{-} \pi^{+(0)} \pi^{-(0)}$ invariant mass distributions are shown in Figs. 1(a) and (b), respectively, where the $\eta^{\prime}$ peak is clearly seen. To ensure that the $\eta^{\prime}$ peak is not from background, an extensive MC study was performed. It was found, as displayed by the hatched histograms in Figs. 1(a) and (b), that none of these background sources produces a peak in the $\pi^{+} \pi^{-} \pi^{+} \pi^{-}$invariant mass spectrum near the $\eta^{\prime}$ mass.

The signal yields are obtained from extended unbinned maximum likelihood fits to the $\pi^{+} \pi^{-} \pi^{+} \pi^{-}$and $\pi^{+} \pi^{-} \pi^{0} \pi^{0}$ invariant mass distributions and the statistical significances for $\eta^{\prime} \rightarrow \pi^{+} \pi^{-} \pi^{+} \pi^{-}$and $\eta^{\prime} \rightarrow \pi^{+} \pi^{-} \pi^{0} \pi^{0}$ are calculated to be $18 \sigma$ and $5 \sigma$, respectively. The branching fractions of $\eta^{\prime} \rightarrow \pi^{+} \pi^{-} \pi^{+(0)} \pi^{-(0)}$ are determined to be $\mathcal{B}\left(\eta^{\prime} \rightarrow \pi^{+} \pi^{-} \pi^{+} \pi^{-}\right)=(8.53 \pm 0.69 \pm 0.64) \times 10^{-5}$ and $\mathcal{B}\left(\eta^{\prime} \rightarrow \pi^{+} \pi^{-} \pi^{0} \pi^{0}\right)=(1.82 \pm 0.35 \pm 0.18) \times 10^{-4}$, which are consistent with the theoretical predictions based on a combination of chiral perturbation theory and vectormeson dominance [7], but not with the broken- $\mathrm{SU}_{6} \times \mathrm{O}_{3}$ quark model [8].

\subsection{Dalitz plot analysis of $\eta \rightarrow \pi^{+} \pi^{-} \pi^{0}$ and} $\eta / \eta^{\prime} \rightarrow \pi^{0} \pi^{0} \pi^{0}[9]$

Considerable theoretical efforts, see e.g. [10-17], have been invested to explain the discrepancy that the predicted 

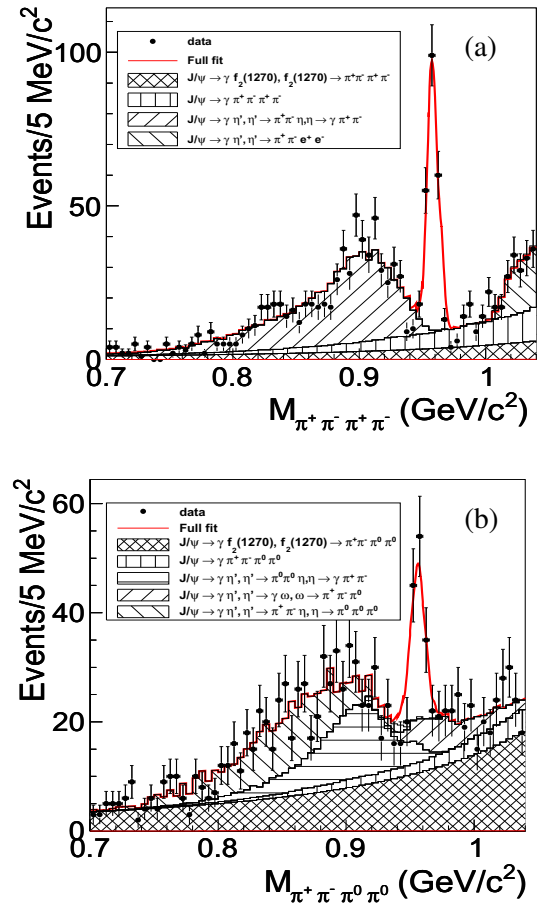

Figure 1. Results of the fits to (a) $M_{\pi^{+} \pi^{-} \pi^{+} \pi^{-}}$and (b) $M_{\pi^{+} \pi^{-} \pi^{0} \pi^{0}}$, where the background contributions are displayed as the hatched histograms.

decay width of $\eta \rightarrow \pi^{+} \pi^{-} \pi^{0}[18]$ at the tree level of ChPT is much lower than the experimental value of $300 \pm 11$ $\mathrm{eV}$ [19]. To distinguish between the different theoretical approaches, precise measurements of the matrix elements for $\eta \rightarrow \pi^{+} \pi^{-} \pi^{0}$ and the decay width are needed.

For $\eta \rightarrow \pi^{+} \pi^{-} \pi^{0}$ decays, a sample of $8 \times 10^{4} \eta \rightarrow$ $\pi^{+} \pi^{-} \pi^{0}$ candidate events are selected and the background contamination is estimated to be about $0.1 \%$ as shown in Fig. 2 (a), which is neglected in the extraction of the Dalitz plot parameters. The two the Dalitz plot variables are defined as $X=\frac{\sqrt{3}}{Q}\left(T_{\pi^{+}}-T_{\pi^{-}}\right)$and $Y=\frac{m_{\eta}+2 m_{\pi}}{m_{\pi}} \frac{T_{\eta}}{Q}-1$, where where $T_{\pi}$ denotes the kinetic energy of a given pion in the $\eta$ rest frame, $Q=m_{\eta}-m_{\pi^{+}}-m_{\pi^{-}}-m_{\pi^{0}}$ is the excess energy of the reaction. The distributions of $\mathrm{X}$ and $\mathrm{Y}$ are shown in Fig. 2 (b) and (c). Using the same parameterization as in Ref. [20], the Dalitz plot parameters for $\eta \rightarrow$ $\pi^{+} \pi^{-} \pi^{0}$ are determined to be $a=-1.128 \pm 0.015 \pm 0.008$, $b=0.153 \pm 0.017 \pm 0.004, d=0.085 \pm 0.016 \pm 0.009$, $f=0.173 \pm 0.028 \pm 0.021$, which are in reasonable agreement with previous measurements.

Figure 3(a) shows the $\pi^{0} \pi^{0} \pi^{0}$ mass spectrum, where the $\eta$ peak is quite clean and the background level is less than $1 \%$. The distribution of the variable $Z$ is displayed in Fig. 3(b). Due to the kinematic boundaries, the interval of $0<Z<0.7$, corresponding to the region of phase space where the $Z$ distribution is flat, is used to extract the slope parameter $\alpha$ from the data. Analogous to the measurement for $\eta \rightarrow \pi^{+} \pi^{-} \pi^{0}$, an unbinned maximum likelihood fit, as displayed in the inset of Fig. 3(b), yields the Dalitz plot slope parameter $\alpha=-0.055 \pm 0.014 \pm 0.004$, which is compatible with the recent results from other experiments and in agreement with the prediction from ChPT at NNLO within two standard deviations of the theoretical uncertainties.

For $\eta^{\prime} \rightarrow \pi^{0} \pi^{0} \pi^{0}$, the $\eta^{\prime}$ signal is also clearly observed in $\pi^{0} \pi^{0} \pi^{0}$ mass spectrum [Fig. 3(c)], where the hatched and shaded histogram show the background contributions estimated from the inclusive $J / \psi$ decays and $\eta^{\prime} \rightarrow \pi^{0} \pi^{0} \eta$, respectively. The Dalitz plot slope parameter for $\eta^{\prime} \rightarrow$ $\pi^{0} \pi^{0} \pi^{0}$ is measured to be $\alpha=-0.640 \pm 0.046 \pm 0.047$ [Fig. 3(d)], which is consistent with but more precise than previous measurements. The value deviates significantly from zero, which implies that final state interactions play an important role in the decay. Up to now, there are just a few predictions about the slope parameter of $\eta^{\prime} \rightarrow \pi^{0} \pi^{0} \pi^{0}$. In Ref. [21], the slope parameter is predicted to be less than 0.03 , which is excluded by our measurement.

\subsection{Observation of $\eta^{\prime} \rightarrow \rho^{ \pm} \pi^{\mp}$ [22]}

With the isobar model, a combined amplitude analysis of the isospin violating decays $\eta^{\prime} \rightarrow \pi^{+} \pi^{-} \pi^{0}$ and $\eta^{\prime} \rightarrow \pi^{0} \pi^{0} \pi^{0}$ is performed. The results shown in the invariant mass spectra of $\pi^{+} \pi^{-}, \pi^{+} \pi^{0}$ and $\pi^{-} \pi^{0}$ (Fig. 4) indicate that significant $P$-wave contribution from $\eta^{\prime} \rightarrow \rho^{ \pm} \pi^{\mp}$ is observed for the first time in $\eta^{\prime} \rightarrow \pi^{+} \pi^{-} \pi^{0}$, which is also clearly indicated as the two clusters in the Dalitz plot of $M_{\pi^{+} \pi^{-}}^{2}$ versus $M_{\pi^{-} \pi^{0}}^{2}$ [Fig. 5 (a) ]. The branching fraction $\mathcal{B}\left(\eta^{\prime} \rightarrow \rho^{ \pm} \pi^{\mp}\right)$ is determined to be $[7.44 \pm 0.60$ (stat $) \pm$ $1.26($ syst $) \pm 1.84($ model $)] \times 10^{-4}$. In addition to the nonresonant $S$-wave, the resonant $\pi-\pi S$-wave with a pole at $(512 \pm 15)-i(188 \pm 12) \mathrm{MeV}$, interpreted as the broad $\sigma$ meson, plays an essential role in the $\eta^{\prime} \rightarrow \pi \pi \pi$ decays. Due to the large interference between non-resonant and resonant $S$-waves, only the sum is used to describe the $S$-wave contribution, and the branching fractions are determined to be $\mathcal{B}\left(\eta^{\prime} \rightarrow \pi^{+} \pi^{-} \pi^{0}\right)_{S}=[37.63 \pm 0.77($ stat $) \pm 2.22($ syst $) \pm$ $4.48($ model $)] \times 10^{-4}$.

For $\eta^{\prime} \rightarrow \pi^{0} \pi^{0} \pi^{0}$, the $P$-wave contribution in two-body rescattering is forbidden by Bose symmetry. The Dalitz plot for $\eta^{\prime} \rightarrow \pi^{0} \pi^{0} \pi^{0}$ is shown in Fig. 5 (b) and the amplitude result is displayed in Fig. 5 (c). The corresponding branching fraction is measured to be $\mathcal{B}\left(\eta^{\prime} \rightarrow \pi^{0} \pi^{0} \pi^{0}\right)=$ $[35.22 \pm 0.82($ stat $) \pm 2.60($ syst $)] \times 10^{-4}$.

The branching fractions of $\eta^{\prime} \rightarrow \pi^{+} \pi^{-} \pi^{0}$ and $\eta^{\prime} \rightarrow$ $\pi^{0} \pi^{0} \pi^{0}$ are in good agreement with and supersede the previous BESIII measurements [23]. The value for $\mathcal{B}\left(\eta^{\prime} \rightarrow\right.$ $\left.\pi^{0} \pi^{0} \pi^{0}\right)$ is two times larger than that from GAMS [(16 \pm $3.2) \times 10^{-4}$ ] [24]. The significant resonant $S$-wave contribution also provides a reasonable explanation for the negative slope parameter of the $\eta^{\prime} \rightarrow \pi^{0} \pi^{0} \pi^{0}$ Dalitz plot [25]. The ratio of the branching fractions between the $S$-wave components $\mathcal{B}\left(\eta^{\prime} \rightarrow \pi^{0} \pi^{0} \pi^{0}\right) / \mathcal{B}\left(\eta^{\prime} \rightarrow \pi^{+} \pi^{-} \pi^{0}\right) S$ is determined as $0.94 \pm 0.029 \pm 0.13$, where the common systematic are cancelled. With the branching fractions of 

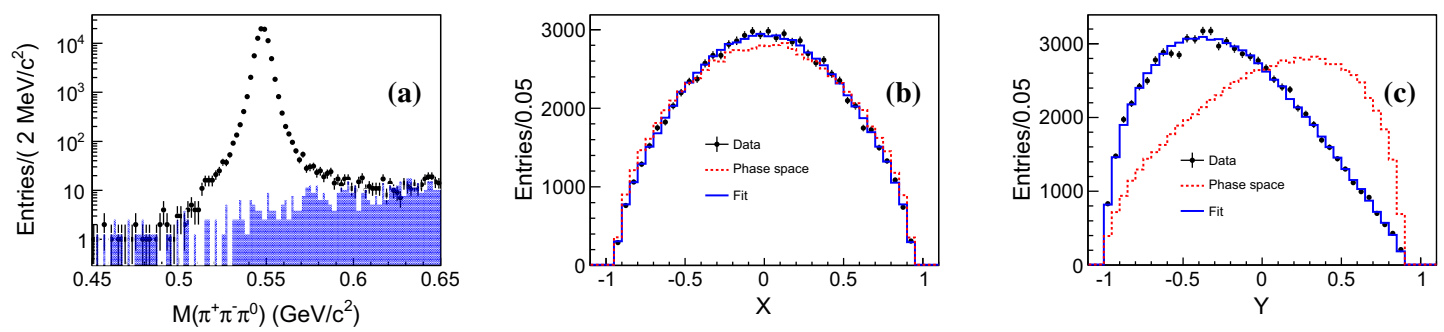

Figure 2. (a) Distribution of $\pi^{+} \pi^{-} \pi^{0}$ invariant mass. Projections of the Dalitz plot as a function of (b) $X$ and (c) $Y$ for $\eta \rightarrow \pi^{+} \pi^{-} \pi^{0}$ obtained from data (dots with error bars) and phase space distributed MC events (dashed line). The result of the fit described in the text (solid line) is also plotted.
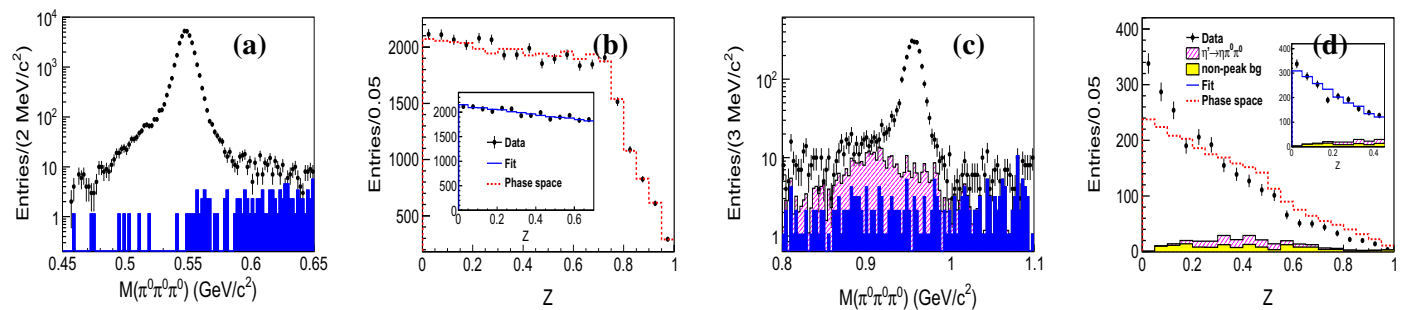

Figure 3. (a) Distribution of $\pi^{0} \pi^{0} \pi^{0}$ invariant mass. (b) Distribution of the kinematic variable $Z$ for $\eta \rightarrow \pi^{0} \pi^{0} \pi^{0}$. (c) Distribution of $\pi^{0} \pi^{0} \pi^{0}$ invariant mass. (d) Distribution of the kinematic variable $Z$ for $\eta^{\prime} \rightarrow \pi^{0} \pi^{0} \pi^{0}$. Dots with error bars are for data, histograms for background contributions, dashed histograms for phase space distributed MC events and the solid lines in the inset are the results of the fit.
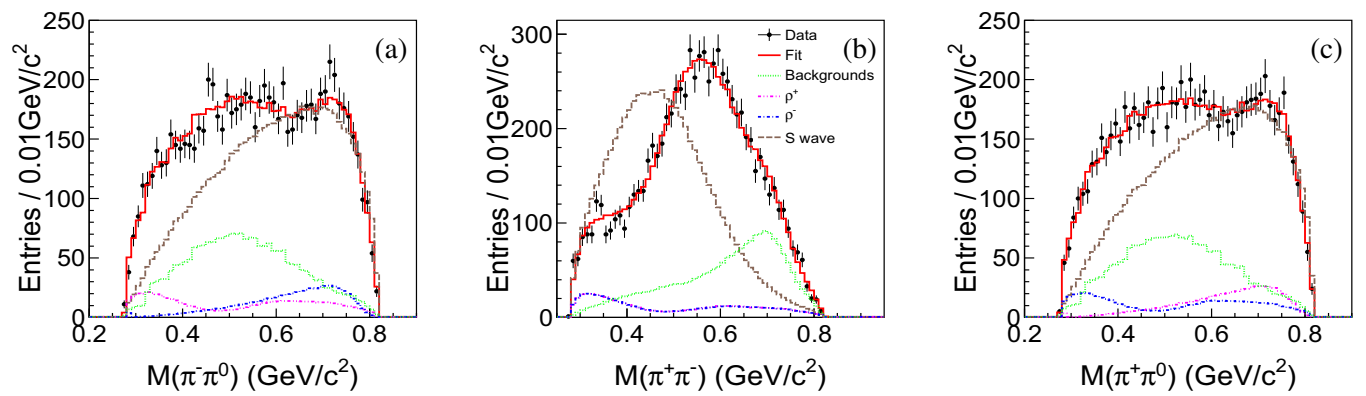

Figure 4. Comparison of the invariant mass distributions of (a) $\pi^{-} \pi^{0}$, (b) $\pi^{+} \pi^{-}$, (c) $\pi^{+} \pi^{0}$ (dots with error bars) and the fit result projections (solid histograms). The dotted, dashed, dash-dotted, and dash-dot-dotted histograms show the contributions from background, $S$ wave, $\rho^{-}$, and $\rho^{+}$, respectively.

$\eta^{\prime} \rightarrow \pi \pi \eta$ taken from PDG [19], $r_{ \pm}=\frac{\mathcal{B}\left(\eta^{\prime} \rightarrow \pi^{+} \pi^{-} \pi^{0}\right)}{\mathcal{B}\left(\eta^{\prime} \rightarrow \pi^{+} \pi^{-} \eta\right)}$ and $r_{0}=\frac{\mathcal{B}\left(\eta^{\prime} \rightarrow \pi^{0} \pi^{0} \pi^{0}\right)}{\mathcal{B}\left(\eta^{\prime} \rightarrow \pi^{0} \pi^{0} \eta\right)}$ are now calculated to be $(8.8 \pm 1.2) \times 10^{-3}$ and $(16.9 \pm 1.4) \times 10^{-3}$, respectively.

\section{$3 \eta / \eta^{\prime}$ radiative decays}

\subsection{Observation of $\eta^{\prime} \rightarrow \gamma e^{+} e^{-}$[26]}

Electromagnetic (EM) Dalitz decays of light pseudoscalar mesons, $P \rightarrow \gamma l^{+} l^{-}\left(P=\pi^{0}, \eta, \eta^{\prime} ; l=\right.$ $e, \mu)$, play an important role in revealing the structure of hadrons and the interaction mechanism between photons and hadrons [27]. In this work, we report the first observation of the Dalitz decay $\eta^{\prime} \rightarrow \gamma e^{+} e^{-}$as shown in Fig. 6 (a) and the branching fraction $\mathcal{B}\left(\eta^{\prime} \rightarrow \gamma e^{+} e^{-}\right)$is measured to be $(4.69 \pm 0.20 \pm 0.23) \times 10^{-4}$.

In addition, we present measurements of the transition form factor (TFF) as a function of $M\left(e^{+} e^{-}\right)$[Figs. 6 (b) and (c) ], which could be described with a single 

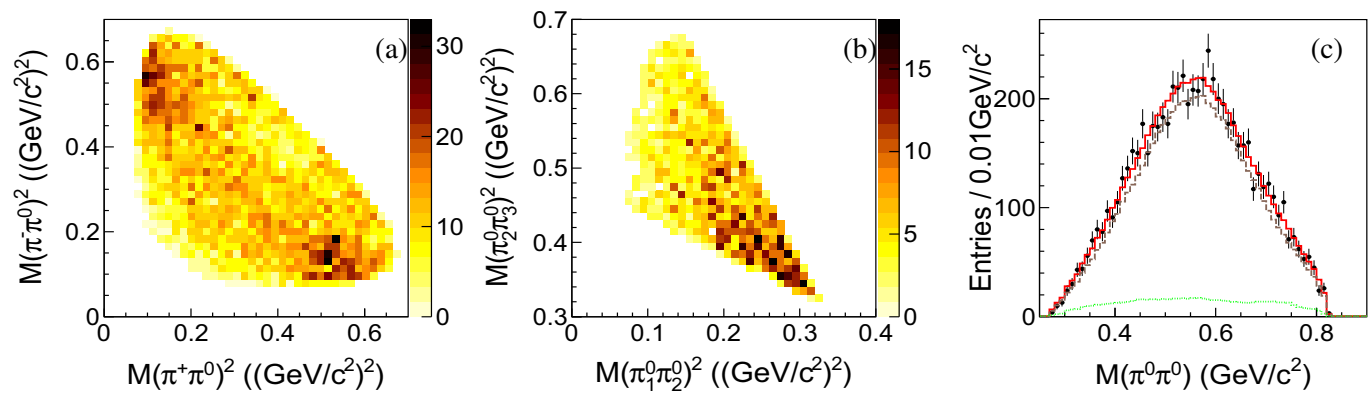

Figure 5. (a) Dalitz plot of $M_{\pi^{+} \pi^{0}}^{2}$ versus $M_{\pi^{-} \pi^{0}}^{2}$ for $\eta^{\prime} \rightarrow \pi^{+} \pi^{-} \pi^{0}$. b) $\eta^{\prime} \rightarrow \pi^{0} \pi^{0} \pi^{0}$ Dalitz plot for candidate events selected from data. (c) Comparison of $\pi^{0} \pi^{0}$ mass spectrum between data (dots with error bars) and the fit result projections (solid histograms).
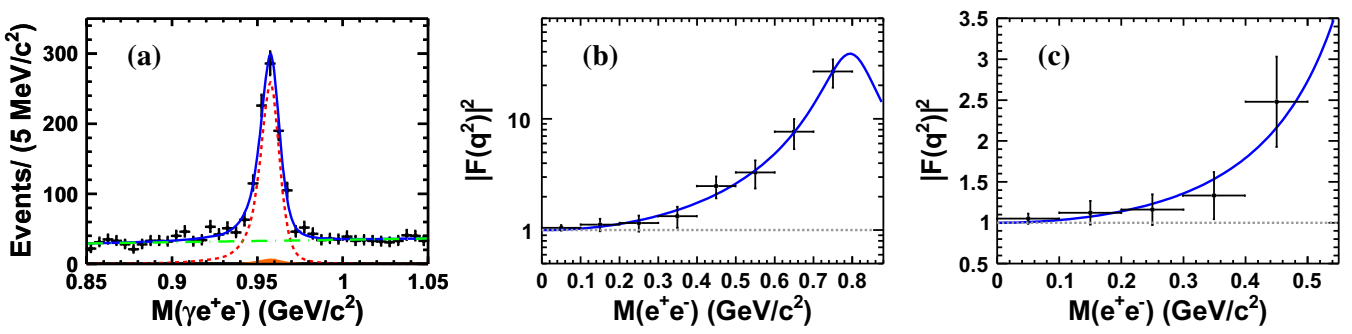

Figure 6. (a) Invariant $\gamma e^{+} e^{-}$mass distribution for the selected signal events. The (black) crosses are the data, the (red) dashed line represents the signal, the (green) dot-dashed curve shows the non-peaking background shapes, the (orange) shaded component is the shape of the peaking background events. (b) Fit to the single pole form factor $|F|^{2}$. (c) Determination of the form factor slope by fitting to $|F|^{2}$.

pole parameterization of $F\left(q^{2}\right)=\frac{1}{\left(1-q^{2} / \Lambda^{2}\right)}$. The mass and width parameters of $\Lambda_{\eta^{\prime}}$ and $\gamma_{\eta^{\prime}}$ are determined to be $(0.79 \pm 0.04 \pm 0.02) \mathrm{GeV}$, and $\gamma_{\eta^{\prime}}=(0.13 \pm 0.06 \pm$ $0.03) \mathrm{GeV}$, respectively. The slope of the TFF corresponds to $(1.60 \pm 0.17 \pm 0.08) \mathrm{GeV}^{-2}$ and agrees within errors with the Vector Meson Dominance (VMD) model. predictions. The uncertainty of the $\eta^{\prime}$ transition form factor slope is in good agreement the best determination in the space-like region from the CELLO collaboration $b_{\eta^{\prime}}=(1.60 \pm 0.16) \mathrm{GeV}^{-2}[28]$, and improves the previous determination of the slope in the time-like region $b_{\eta^{\prime}}=(1.7 \pm 0.4) \mathrm{GeV}^{-2}[27,29]$.

\subsection{Observation of $\eta^{\prime} \rightarrow e^{+} e^{-} \omega$ [30]}

In 2013 BESIII reported the measurement of $\eta^{\prime} \rightarrow$ $\pi^{+} \pi^{-} e^{+} e^{-}$[31], which is found to be dominated by $\eta^{\prime}$ $\rightarrow \rho e^{+} e^{-}$, in agreement with theoretical predictions [32?]. Based on theoretical models [32, 34], the branching fraction of $\eta^{\prime} \rightarrow \omega e^{+} e^{-}$is predicted to be around $2.0 \times 10^{-4}$, but until now there has been no measurement of this decay.

The scatter plot of $M\left(\pi^{0} \pi^{+} \pi^{-} e^{+} e^{-}\right)$versus $M\left(\pi^{0} \pi^{+} \pi^{-}\right)$ is shown in Fig. 7 (a), where the cluster in the $\eta^{\prime}$ and $\omega$ region corresponds to the decay $\eta^{\prime} \rightarrow \omega e^{+} e^{-}$. The $\eta^{\prime}$ peak is clearly seen in the distributions of $M\left(\pi^{0} \pi^{+} \pi^{-} e^{+} e^{-}\right)$ shown in Fig. 7 (b). To improve the mass resolution, the fit to $M\left(\pi^{0} \pi^{+} \pi^{-} e^{+} e^{-}\right)-M\left(\pi^{0} \pi^{+} \pi^{-}\right)$is performed to extract the signal yields as indicated in Fig. 7 (c). For the first time, the decay of $\eta^{\prime} \rightarrow \omega e^{+} e^{-}$is observed with a statistical significance of $8 \sigma$, and its branching fraction is measured to be $\mathcal{B}\left(\eta^{\prime} \rightarrow \omega e^{+} e^{-}\right)=[1.97 \pm$ 0.34 (stat) \pm 0.17 (syst) $] \times 10^{-4}$, which is consistent with theoretical prediction, $2.0 \times 10^{-4}$ [32].

\subsection{Observation of $\eta^{\prime} \rightarrow \gamma \gamma \pi^{0}$ [35]}

Most recently the observation of $\eta^{\prime} \rightarrow \gamma \gamma \pi^{0}$ was reported by the BESIII Collaboration. Figure 8(a) shows the $\gamma \gamma \pi^{0}$ invariant mass spectrum, where the clear $\eta^{\prime}$ peak is observed. Assuming that the inclusive decay $\eta^{\prime} \rightarrow \gamma \gamma \pi^{0}$ can be attributed to the vector mesons $\rho$ and $\omega$ and the non-resonant contribution, we apply a fit to the $\gamma \pi^{0}$ invariant mass [Fig. 8 (b)] to determine the branching fraction for the non-resonant $\eta^{\prime} \rightarrow \gamma \gamma \pi^{0}$ decay using the $\eta^{\prime}$ signal events with $\left|M_{\gamma \gamma \pi^{0}}-m_{\eta^{\prime}}\right|<25 \mathrm{MeV} / \mathrm{c}^{2}$. In the fit, the $\rho$ $\omega$ interference is considered, but possible interference between the $\omega(\rho)$ and the non-resonant process is neglected.

The branching fraction of the inclusive decay is measured for the first time to be $\mathcal{B}\left(\eta^{\prime} \rightarrow \gamma \gamma \pi^{0}\right)_{\text {Incl. }}=(3.20 \pm$ $0.07 \pm 0.23) \times 10^{-3}$, which is much lower than that of the theoretical predictions[36, 37]. In addition, the branching fraction for the non-resonant decay is determined to be 

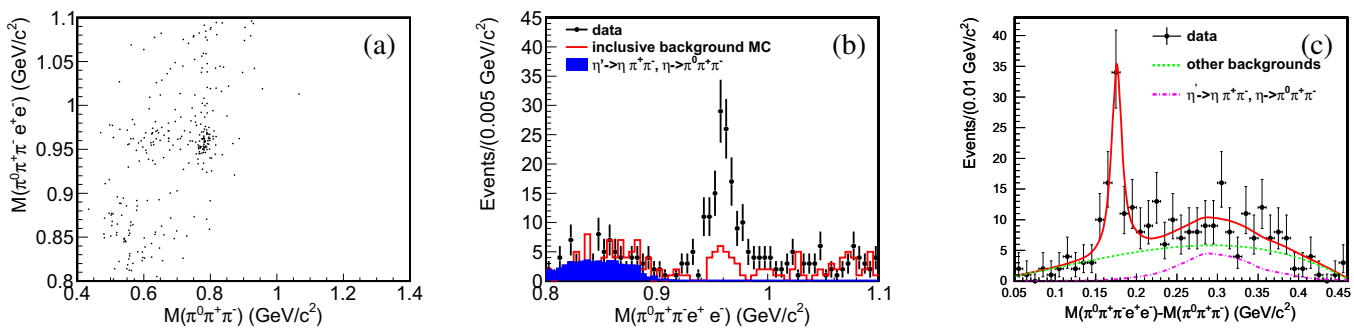

Figure 7. (a) Distribution of $M\left(\pi^{0} \pi^{+} \pi^{-} e^{+} e^{-}\right)$versus $M\left(\pi^{0} \pi^{+} \pi^{-}\right)$; Distribution of $M\left(\pi^{0} \pi^{+} \pi^{-} e^{+} e^{-}\right)-M\left(\pi^{0} \pi^{+} \pi^{-}\right)$and the fit results. The dots with error bars are for data, the histograms or dash-dotted line are for background contributions and the dotted curve shows the background except for the background events from $\eta^{\prime} \rightarrow \pi^{+} \pi^{-} \eta$.
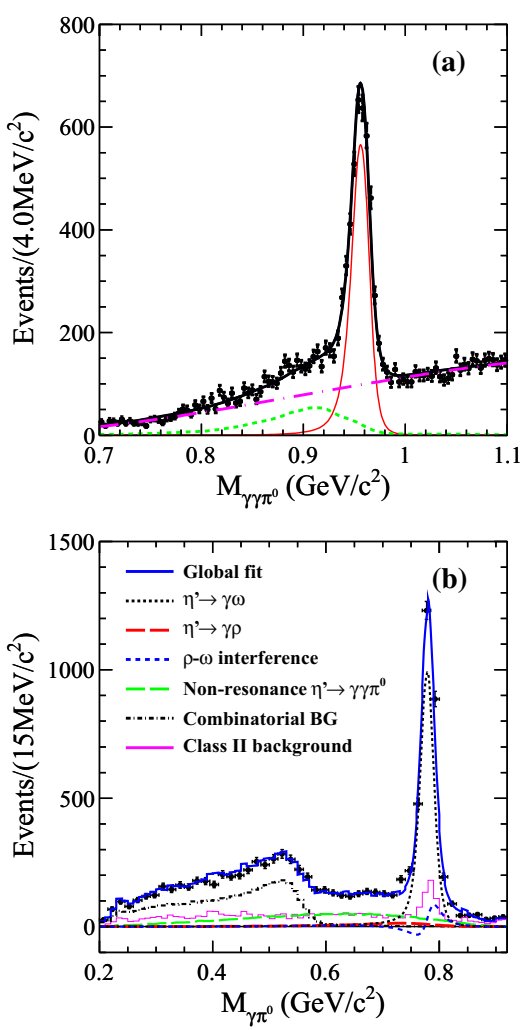

Figure 8. (a) Distribution of $M_{\gamma \gamma \pi^{0}}$ for the selected inclusive $\eta^{\prime} \rightarrow \gamma \gamma \pi^{0}$ signal events via $J / \psi \rightarrow \gamma \eta^{\prime}$ decay. The dots with error bars are for data, the dotted and dot-dashed curve are the shapes of the background events. The total fit result is shown as the solid line. (b) Distribution of the invariant mass $M_{\gamma \pi^{0}}$ and fit results. The dots with error bars are data; the dotted-curve is from the $\omega$-contribution; the long dashed-curve is from the $\rho$-contribution; the short dashed-curve is the contribution of $\rho$ $\omega$ interference; the long dashed curve is the non-resonance; the histogram is from the background events; the short dot-dashed curve is the combinatorial backgrounds of $\eta^{\prime} \rightarrow \gamma \omega, \gamma \rho$. The solid line shows the total fit function.
$\mathcal{B}\left(\eta^{\prime} \rightarrow \gamma \gamma \pi^{0}\right)_{\mathrm{NR}}=(6.16 \pm 0.64 \pm 0.67) \times 10^{-4}$, which agrees with the upper limit measured by the GAMS-2000 experiment [38]. As a validation of the fit, the product branching fraction with the omega intermediate state involved is obtained to be $\mathcal{B}\left(\eta^{\prime} \rightarrow \gamma \omega\right) \cdot \mathcal{B}\left(\omega \rightarrow \gamma \pi^{0}\right)=$ $(2.37 \pm 0.07 \pm 0.18) \times 10^{-3}$, which is consistent with the PDG value [5]. These results are useful to test QCD calculations on the transition form factor, and provide valuable inputs to the theoretical understanding of the light meson decay mechanisms.

\subsection{Preliminary results : study of $\eta^{\prime} \rightarrow \gamma \pi^{+} \pi^{-}$decay dynamics}

Theoretically the non-resonant part of coupling in $\eta^{\prime} \rightarrow \gamma \pi^{+} \pi^{-}$is accounted for by the higher term of WessZumino-Witten (WZW) ChPT Lagrangian (also known as the box anomaly). However the decay dynamics of $\eta^{\prime} \rightarrow \gamma \pi^{+} \pi^{-}$has been explored with very limited statistics only and new measurements are needed to clarify the scenario. In this work, a sample of $9 \times 10^{5} \eta^{\prime} \rightarrow \gamma \pi^{+} \pi^{-}$events is selected to investigate its decay dynamics and the $\pi^{+} \pi^{-}$ mass spectrum is shown in Fig. 9 with a background level of $1 \%$.

The shape of the $M_{\pi^{+} \pi^{-}}$spectrum is analyzed using parameterization relying on model-dependent and modelindependent approaches respectively. For the modeldependent approach, the results show that only $\rho(770)$ resonance is insufficient to describe the data even if one takes into account both $\rho(770)$ and omega resonances and the interference between them. The fit performance gets much better after including the box anomaly [Fig. 9 (a)] with a statistical significance larger than $37 \sigma$. We also try to replace the box anomaly with $\rho(1450)$ [Fig. 9 (b)] by fixing its mass the width to be the world average values, the fit can also provide a reasonable description of data. Therefore, we conclude that in addition to $\rho(770)$ and $\omega$, the box anomaly is necessary, but the contribution from $\rho(1450)$ could not be ruled out.

With the inclusion of $\omega$ into the expansion, $M_{\pi^{+} \pi^{-}}$spectrum is very well described with the model-independent parameterisation [39] as shown in Fig. 9 (c), the values of parameters being $\alpha=0.992 \pm 0.039 \pm 0.067$ 

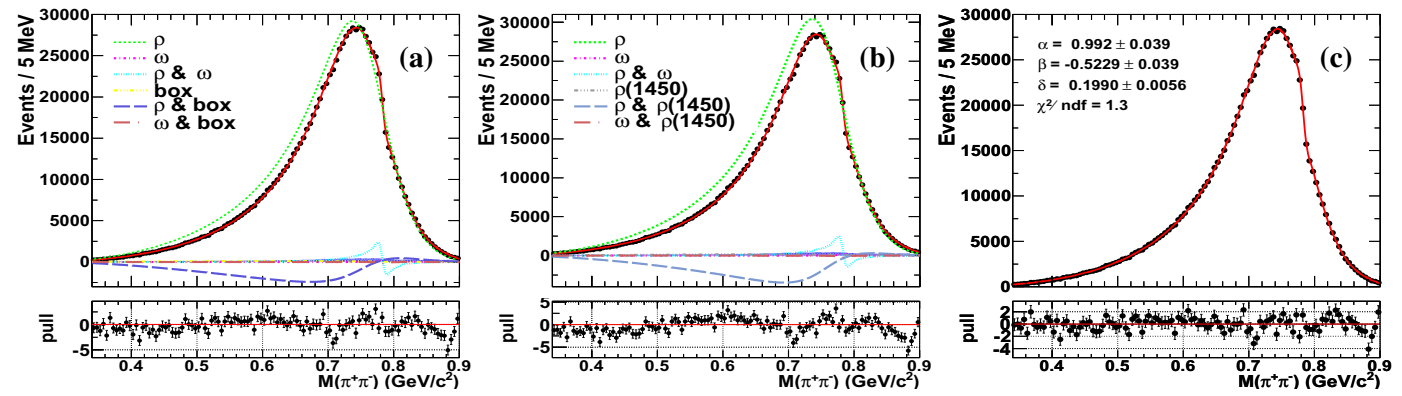

Figure 9. The results of the model-dependent fits to $M_{\pi \pi}$ with (a) $\rho-\omega-$ box anomaly and (b) $\rho-\omega-\rho$ (1450). (c) The results of model-independent fit with $\omega$ interference.

$\mathrm{GeV}^{-2}, \beta=-0.523 \pm 0.039 \pm 0.066 \mathrm{GeV}^{-4}, \delta=$ $0.199 \pm 0.006 \pm 0.010$. The value of $\alpha$ found in this work is compatible with the work of [39].

\section{Search for rare decays of $\eta^{\prime} \rightarrow K^{ \pm} \pi^{\mp}$ [40]}

Non-leptonic weak decays are valuable tools for exploring physics beyond the Standard Model. Among nonleptonic decays, the decay of the light pseudoscalar meson $\eta^{\prime} \rightarrow K^{ \pm} \pi^{\mp}$ is interesting because it is fundamental to understand the long-standing problem of the $\Delta I=1 / 2$ rule in weak non-leptonic interactions. The branching fraction of $\eta^{\prime} \rightarrow K^{ \pm} \pi^{\mp}$ decay is predicted to be of the order of $10^{-10}$ or higher [41], with a large long-range hadronic contribution expected.

A search for the non-leptonic weak decay $\eta^{\prime} \rightarrow K^{ \pm} \pi^{\mp}$ is performed for the first time through the $J / \psi \rightarrow \phi \eta^{\prime}$ decay, while no evidence for $\eta^{\prime} \rightarrow K^{ \pm} \pi^{\mp}$ is seen. Thus the $90 \%$ C.L. upper limit on $\mathcal{B}\left(\eta^{\prime} \rightarrow K^{ \pm} \pi^{\mp}\right)$ is calculated to be $3.8 \times 10^{-5}$.

\section{Summary}

In summary the recent results on $\eta / \eta^{\prime}$ decays at BESIII are presented with a sample of $1.3 \times 10^{9} \mathrm{~J} / \psi$ events. The hadronic decays of $\eta^{\prime} \rightarrow \pi^{+} \pi^{-} \pi^{+} \pi^{-}$and $\eta^{\prime} \rightarrow \pi^{+} \pi^{-} \pi^{0} \pi^{0}$ are also observed and the measurement branching fractions are consistent with the theoretical predictions; The Dalitz plots of $\eta \rightarrow \pi^{+} \pi^{-} \pi^{0}$ and $\eta / \eta^{\prime} \rightarrow \pi^{0} \pi^{0} \pi^{0}$ are analyzed and the corresponding matrix elements are extracted; The observation of $\eta^{\prime} \rightarrow \rho^{\mp} \pi^{ \pm}$is reported for the first time; the new decay mode of $\eta^{\prime} \rightarrow \gamma e^{+} e-$ is also observed and the TFF is measured with the function of $M_{e^{+}} e^{-}$; the decay dynamics of $\eta^{\prime} \rightarrow \gamma \pi^{+} \pi^{-}$is investigated with both model-dependent and model-independent parameterizations. It is found that the extra contribution is necessary to describe data besides the contributions from $\rho(770)$ and $\omega$. The above results indicate that there is a rich field to be explored in $\eta / \eta^{\prime}$ physics, which motivates to search for their rare and forbidden decays.

Based on the roughly estimation, the available $1.3 \times 10^{9}$ $J / \psi$ events enables to investigate the Dalitz plot of $\omega \rightarrow$
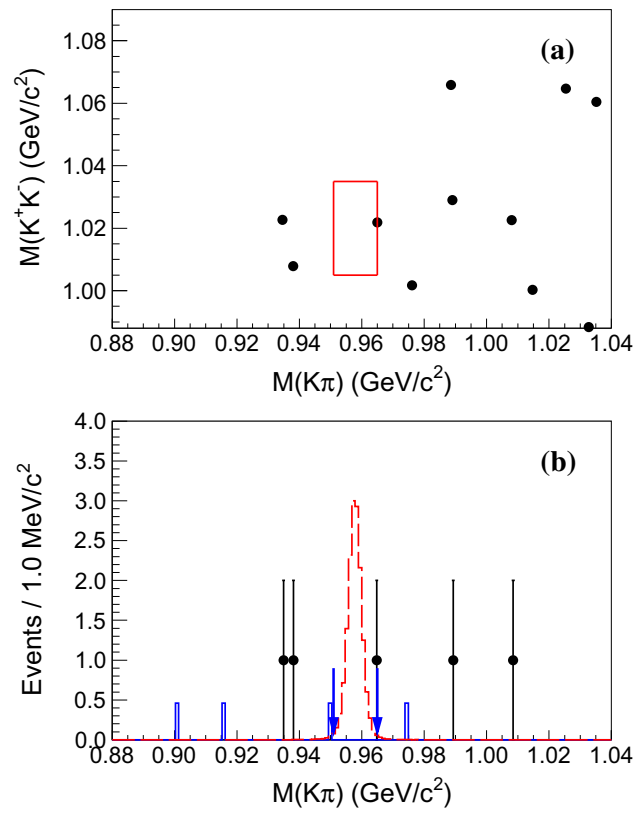

Figure 10. (a) Scatter plot of $M\left(K^{+} K^{-}\right)$versus $M\left(K^{ \pm} \pi^{\mp}\right)$, where the box indicates the signal region with $\left|M\left(K^{+} K^{-}\right)-M(\phi)\right|<$ $15 \mathrm{MeV} / c^{2}$ and $\left|M\left(K^{ \pm} \pi^{\mp}\right)-M\left(\eta^{\prime}\right)\right|<7 \mathrm{MeV} / c^{2}$. (b) The $K^{ \pm} \pi^{\mp}$ invariant mass distribution, where the arrows show the signal region. The dots with error bars are for data, the dashed histogram is for the signal MC with arbitrary normalization, and the solid histogram is the background contamination from a MC simulation of $J / \psi \rightarrow \phi \pi^{+} \pi^{-}$.

$\pi^{+} \pi^{-} \pi^{0}$, which could be used to test the recent theoretical calculations based on the dispersive theory. This analysis is undergoing and the preliminary results will be released soon. However it is still not sufficient to search for $\omega$ rare decays. Hopefully a sample of 10 billion $J / \psi$ events will be collected at the BESIII detector, which allows the study of light meson decays, such as $\eta / \eta^{\prime}$ and $\omega$, with unprecedented precision. 


\section{References}

[1] F. Niecknig, B. Kubis, S. P. Schneider, Eur. Phys. J. C 72,2014 (2012).

[2] I. V. Danilkin, C. Fernndez-Ramrez et al., Phys. Rev. D 91,094029 (2015).

[3] A. Aloisio et al. (KLOE Collaboration), Phys. Lett. B 561 (2003).

[4] M. Ablikim et al. (BESIII Collaboration), Chin. Phys. C 36, 915 (2012) ; M. Ablikim et al. (BESIII Collaboration), arXiv :1607.00738 [hep-ex].

[5] C. Patrignani et al. (Particle Data Group), Chin. Phys. C 40, 100001 (2016).

[6] M. Ablikim et al. (BESIII Collaboration), Phys. Rev. Lett. 112, 251801 (2014).

[7] F. K. Guo, B. Kubis and A. Wirzba, Phys. Rev. D 85, 014014 (2012).

[8] D.Parashar, Phys. Rev. D 19, 268 (1979).

[9] M. Ablikim et al. (BESIII Collaboration), Phys. Rev. D 92, 012014 (2015).

[10] A. Anisovich and H. Leutwyler, Phys. Lett. B 375, 335 (1996).

[11] S. P. Schneider, B. Kubis, and C. Ditsche, JHEP 1102, 028 (2011).

[12] J. Gasser and H. Leutwyler, Nucl. Phys. B 250, 539 (1985).

[13] J. Kambor, C. Wiesendanger and D. Wyler,Nucl. Phys. B 465, 215 (1996).

[14] J. Bijnens and K. Ghorbani, JHEP 0711, 030 (2007).

[15] K. Kampf, M. Knecht, J. Novotny and M. Zdrahal, Phys. Rev. D 84, 114015 (2011).

[16] G. Colangelo, S. Lanz, H. Leutwyler and E. Passemar, PoS EPS -HEP2011, 304 (2011).

[17] P. Guo et al., Phys. Rev. D 92, 054016 (2015).

[18] H. Osborn and D. J. Wallace, Nucl. Phys. B 20, 23 (1970) ; J. A. Cronin, Phys. Rev. 161, 1483 (1967).

[19] K. A. Olive et al. (Particle Data Group), Chin. Phys. C 38, 090001 (2014).

[20] F. Ambrosino et al. (KLOE Collaboration), JHEP 0805, 006 (2008).
[21] C. Roiesnel, T. N. Truong, Paris Ec. Polytech. A 515, 0982(1982).

[22] M. Ablikim et al. (BESIII Collaboration), Phys. Rev. Lett. 118, 012001 (2017).

[23] M. Ablikim et al. (BESIII Collaboration), Phys. Rev. Lett. 108, 182001 (2012).

[24] D. Alde, F. G. Binon, and C. Bricman, Z. Phys. C 36, 603 (1987).

[25] M. Ablikim et al. (BESIII Collaboration), Phys. Rev. D 92, 012014 (2015).

[26] M. Ablikim et al. (BESIII Collaboration), Phys. Rev. D 83, 012003 (2011).

[27] L. G. Landsberg, Phys. Rept. 128, 301 (1985).

[28] H. J. Behrend et al. (CELLO Collaboration), Z. Phys. C 49, 401 (1991).

[29] R. I. Dzhelyadin et al., Phys. Lett. B 88, 379 (1979).

[30] M. Ablikim et al., (BESIII Collaboration), Phys. Rev. D 92, 051101(R) (2015).

[31] M. Ablikim et al., (BESIII Collaboration), Phys. Rev. D 87, 092011 (2013).

[32] A. Faessler, C. Fuchs, M. Krivoruchenko, Phys. Rev. C 61, 035206 (2000).

[33] B. Borasoy, R. Nissler, Eur. Phys. J. A 33, 95 (2007).

[34] C. Terschlüsen, S. Leupold, M. Lutz, Eur. Phys. J. A 48, 190 (2012).

[35] M. Ablikim et al., (BESIII Collaboration), arXiv :1612.0572 [hep-ex].

[36] R. Jora, Nucl. Phys. Proc. Suppl. 207-208, 224 (2010).

[37] R. Escribano, PoS QNP 2012, 079 (2012).

[38] D. Alde et al. (GAMS-2000 Collaboration), Z. Phys. C 36, 603 (1987).

[39] F. Stollenwerk, C. Hanhart, A. Kupsc, U.-G. Meissner, A. Wirzba, Phys. Lett. B707, 184 (2012).

[40] M. Ablikim et al., (BESIII Collaboration), Phys. Rev. D 93, 072008 (2016).

[41] L. Bergström and H. R. Rubinstein, Phys. Lett. B 203, 183 (1988).

[42] D. M. Asner et al., Int. J. Mod. Phys. A 24, 1 (2009). 